\title{
ANALYSIS AND MEASUREMENT OF COUPLING EFFECTS IN THE TRANSFER LINE FROM PS TO SPS FOR THE LHC PROTON BEAM
}

\author{
G. Arduini, Y. Chao*, M. Giovannozzi, J. Klem, D. Manglunki, M. Martini \\ CERN, Geneva, Switzerland
}

\begin{abstract}
The tight emittance budget for injection into the LHC demands an accurate matching of the transfer line from the PS to the SPS to minimise the injection blow-up. Precise two-dimensional beam profile measurements with Optical Transition Radiation (OTR) screens have recently pointed towards the presence of coupling in the LHC beam transfer. The new algorithms developed to analyse the profile data from the OTR screens and to quantify the observed coupling (in particular the determination of the complete $5 \times 5$ beam covariance matrix) are discussed. The results of the measurements and their dependence on the extraction conditions in the PS (trajectory and momentum) are presented and discussed in detail.
\end{abstract}

\section{INTRODUCTION}

As an important link in the LHC injection chain, the transfer of proton beam from the PS to the SPS through the lines TT2 and TT10 will need to be executed with high precision.

During the 1999 run [1] 2D beam profile measurements performed with OTR screens in TT10 revealed the presence of beam cross-plane coupling that was not due to the dispersion pattern in the line. Furthermore the r.m.s. projected beam sizes at the OTR's were found to be inconsistent. A more refined treatment of the OTR data and the study of the observed coupling were therefore pursued in 2000 [2].

Trajectory difference measurements were performed to verify the optics with complete phase-space coverage. Trajectory oscillations were generated with horizontal and vertical dipoles in TT2 and the responses recorded at 7 beam position monitors in TT10. The in-plane agreement between measurement and model is remarkable while the cross-plane trajectory oscillation amplitude is of the order of $5 \%$ of the in-plane trajectory oscillation amplitude. This coupling, albeit small, is unmistakable in the sense that normalised correlations between the in-plane and cross-plane trajectories exceed $90 \%$ in all cases analysed. The zero crossing in the back-propagated trajectories in the cross plane should reveal possible sources of coupling. However a single zero crossing point common to all cross-plane trajectories could not be identified.

The trajectory difference technique was also employed to quantify the coupling induced by stray fields in the region between the PS extraction and the beginning of TT2. Both the PS extraction bumpers and septum were used to generate trajectory oscillations. The cross-plane trajectory induced in this region is below $2 \%$.

\section{BEAM COVARIANCE MATRIX MEASUREMENT}

\subsection{Method}

The independent parameters to be extracted from each OTR 2D profile are the $\langle\mathbf{X X}\rangle,\langle\mathbf{X Y}\rangle$ and $\langle\mathbf{Y Y}\rangle$ correlations. They are functions of the $5 \times 5$ beam covariance matrix at an arbitrary point $\mathbf{P}$, and the transport optics encompassing the OTR region and $\mathbf{P}$. If the latter is known with confidence, then with a sufficient number of OTR's and/or different optics, one can solve for the beam covariance matrix at $\mathbf{P}$.

If the dispersion is well measured at the OTR's and there is no intrinsic transverse-longitudinal correlation, then one can subtract all dispersive components in the beam covariance and proceed to solve only for the $4 \times 4$ covariance matrix and the momentum spread. If known, the momentum spread can be used to further constrain the fit. Therefore there are 4 options (with increasing redundancy) for the fitting configuration:

- 15-parameter fit: all 15 independent beam covariance matrix elements are used as fitting parameters.

- 14-parameter fit: same as the above but with the momentum spread imposed as a constraint.

- 11-parameter fit: well-known dispersion and no additional transverse-longitudinal correlation are assumed.

- 10-Parameter fit: same as the above but with momentum spread imposed as a constraint.

In all cases the number of OTR-based constraints in the fit is always given by $3 \times$ Notr $\times$ Noptics where Notr and Noptics are the number of OTR's and optics, respectively.

\subsection{Robustness of existing OTR configuration}

An analysis based on Singular Value Decomposition (SVD) was performed on the existing OTR configuration to evaluate the degree of independence of the signals and to identify possible weak signals or nearly irresolvable combinations. It turned out that no singular combination is present. This provides enhanced confidence in the ensuing OTR measurement and analysis.

\subsection{Results for the LHC transfer at $26 \mathrm{GeV} / \mathrm{C}$}

The described method has been applied to the LHC transfer using the data provided by the 4 OTR monitors

\footnotetext{
* On leave from Thomas Jefferson National Accelerator Facility, Newport News, VA USA.
} 
installed in TT10. Three different optical settings of the TT10 part were used in each experiment to obtain enough constraints required for the application of the 4 fitting methods mentioned in Section 1, with extra redundancy. The beam covariance matrix can be determined only at those points upstream of the region where the optics is changed. Table 1 shows the measured beam covariance matrices. In the diagonal are displayed the $\sigma$ 's of $X, X$, $\mathrm{Y}, \mathrm{Y}^{\prime}$, and $\Delta \mathrm{P}$ in units of $\mathrm{m}$, radian or unity, while in the off-diagonal elements the normalised cross-correlations (in the range $[-1,1]$ ) are displayed.

Table 1: Normalised Covariance Matrix at the beginning of TT10 for various fitting modes for the LHC transfer

\begin{tabular}{|c|c|c|c|c|c|}
\hline $\mathrm{X}$ & $\mathrm{X}^{\prime}$ & $\mathrm{Y}$ & $\mathrm{Y}^{\prime}$ & $\Delta \mathrm{P}$ & \\
\hline 0.002 & -0.843 & 0.444 & 0.264 & 0.040 & $\mathrm{X}$ \\
\cline { 2 - 6 } & 0.0001 & -0.310 & -0.056 & -0.091 & $\mathrm{X}^{\prime}$ \\
\cline { 2 - 6 } & 0.0007 & 0.805 & 0.416 & $\mathrm{Y}$ \\
\cline { 2 - 6 } & & 0.00005 & 0.070 & $\mathrm{Y}^{\prime}$ \\
\cline { 2 - 6 } & 15-parameter fit & 0.0002 & $\Delta \mathrm{P}$ \\
\cline { 3 - 5 }
\end{tabular}

\begin{tabular}{|c|c|c|c|c|c|}
\hline $\mathrm{X}$ & $\mathrm{X}^{\prime}$ & $\mathrm{Y}$ & $\mathrm{Y}^{\prime}$ & $\Delta \mathrm{P}$ & \\
\hline 0.002 & -0.838 & 0.417 & 0.275 & -0.088 & $\mathrm{X}$ \\
\hline \multirow{6}{*}{} & 0.0001 & -0.285 & -0.067 & 0.031 & $\mathrm{X}^{\prime}$ \\
\cline { 2 - 6 } & 0.0007 & 0.817 & 0.439 & $\mathrm{Y}$ \\
\hline \multicolumn{7}{|c|}{0.00005} & 0.156 & $\mathrm{Y}^{\prime}$ \\
\hline \multicolumn{1}{|c|}{ 14-parameter fit $\left(\sigma_{\mathbf{p}}=\mathbf{0 . 0 0 0 1 5}\right)$} & & $\Delta \mathrm{P}$ \\
\hline
\end{tabular}

\begin{tabular}{|c|c|c|c|c|c|}
\hline $\mathrm{X}$ & $\mathrm{X}^{\prime}$ & $\mathrm{Y}$ & $\mathrm{Y}^{\prime}$ & $\Delta \mathrm{P}$ & \\
\hline 0.002 & -0.846 & 0.459 & 0.265 & & $\mathrm{X}$ \\
\cline { 3 - 6 } & 0.0001 & -0.292 & -0.057 & & $\mathrm{X}^{\prime}$ \\
\cline { 2 - 6 } & 0.0006 & 0.843 & & $\mathrm{Y}$ \\
\cline { 2 - 6 } & & 0.00005 & & $\mathrm{Y}^{\prime}$ \\
\cline { 3 - 6 } & & & 0.00006 & $\Delta \mathrm{P}$ \\
\cline { 3 - 5 }
\end{tabular}

\begin{tabular}{|c|c|c|c|c|c|}
\hline $\mathrm{X}$ & $\mathrm{X}^{\prime}$ & $\mathrm{Y}$ & $\mathrm{Y}^{\prime}$ & $\Delta \mathrm{P}$ & \\
\hline 0.002 & -0.842 & 0.454 & 0.255 & & $\mathrm{X}$ \\
\hline \multirow{7}{*}{} & 0.0001 & -0.264 & -0.041 & & $\mathrm{X}^{\prime}$ \\
\cline { 2 - 5 } & 0.0006 & 0.889 & & $\mathrm{Y}^{\prime}$ \\
\hline \multicolumn{7}{|c|}{0.00005} & & $\mathrm{Y}^{\prime}$ \\
\cline { 2 - 5 } & &
\end{tabular}

A few observations can be made:

- The fit quality is quite good in all cases. Reproducibility of measurement is good for the transverse components, important for resolving coupled degrees of freedom.

- A nontrivial amount of beam coupling can be seen in all cases.

- The momentum spread obtained from the 11parameter fit is about a factor of 2 smaller than the value measured in the PS machine, but the latter is obtained by assuming a certain bunch shape whereas in the OTR-based fitting, no bunch shape was assumed. The discrepancy becomes smaller when the fit is relaxed to allow for intrinsic transverselongitudinal correlation.
The reproducibility of the measurements is also demonstrated in Table 2, listing the Twiss parameters derived from 3 sets of data, each analysed by the 4 different modes of fitting described in Section 2.1. No significant scatter in the data is observed. Measured r.m.s. emittances in TT10 are also presented.

Table 2: Twiss parameters and emittance

\begin{tabular}{|c|c|c|c|c|}
\hline & $\beta_{\mathrm{H}}[\mathrm{m}]$ & $\alpha_{\mathrm{H}}$ & $\beta_{\mathrm{v}}[\mathrm{m}]$ & $\alpha_{\mathrm{v}}$ \\
\hline Meas. & $40.2 \pm 1.5$ & $1.58 \pm 0.04$ & $22.8 \pm 1.2$ & $-1.5 \pm 0.2$ \\
\hline Model & 31.38 & 0.749 & 18.729 & -0.88 \\
\hline$\varepsilon_{\text {OTR }}[\mu \mathrm{m}]$ & \multicolumn{2}{|c|}{$0.111 \pm 0.003$} & \multicolumn{2}{|c|}{$0.020 \pm 0.003$} \\
\hline
\end{tabular}

With the results obtained from the trajectory difference measurement, one can construct an empirical transfer matrix between any pair of points in TT2/TT10. Its ondiagonal components are those of the model, since we are confident of its correctness as discussed in Section 1, while its off-diagonal components are determined from the measurements described in the same section. The comparison between the beam sizes measured at the TT2 Secondary Emission Monitors (SEM-grids MSG257, MSG267, and MSG277), and those back-propagated from the OTR's (see Table 3) shows a good agreement with the exception of the horizontal (vertical) size at MSG257 (MSG277) where discrepancies of about 30\% are observed. The difference might come from the different resolution of the instruments and the different methods used to calculate the r.m.s. beam size.

Table 3: Comparison between MSG-derived $\sigma$ 's and those back-propagated from TT10 OTR

\begin{tabular}{|l|c|c|c|c|}
\hline & \multicolumn{2}{|c|}{$\sigma_{\mathrm{H}}[\mathrm{mm}]$} & \multicolumn{2}{c|}{$\sigma_{\mathrm{v}}[\mathrm{mm}]$} \\
\hline & Meas. & Prop. & Meas. & Prop. \\
\hline MSG257 & 1.34 & 1.01 & 1.03 & 0.91 \\
\hline MSG267 & 1.29 & 1.34 & 0.84 & 0.83 \\
\hline MSG277 & 1.87 & 1.75 & 0.91 & 0.62 \\
\hline
\end{tabular}

\subsection{Search of coupling sources}

If the back-propagated beam covariance matrix is tabulated by element index and there exists a localized source $\mathbf{S}$ of coupling, then the following functions should vanish on both sides of $\mathbf{S}$

$$
\begin{aligned}
& \frac{\sigma_{23}}{\sigma_{14}}-\frac{\sigma_{33}}{\sigma_{11}}, \frac{-\sigma_{12} \sigma_{23}+\sigma_{24} \sigma_{33}-\sigma_{23} \sigma_{34}}{\sigma_{33}}, \\
& \frac{-\sigma_{12} \sigma_{14}+\sigma_{24} \sigma_{11}-\sigma_{14} \sigma_{34}}{\sigma_{11}}, \frac{\sigma_{13}^{2}}{\sigma_{11} \sigma_{33}},
\end{aligned}
$$

and the vectors $\mathrm{v}_{\mathrm{C}}=\left(\sigma_{23}, \sigma_{14}, \sigma_{24}, \sigma_{13}\right), \mathrm{m}_{\mathrm{C}}=\left(\sigma_{33}, \sigma_{11}\right.$, $\left.\sigma_{12}+\sigma_{34}, 0\right)$ should be linearly dependent, i.e. the $2 \times 4$ matrix $M_{C}=\left(v_{C}, m_{C}\right)$ should be degenerate. Then, the coefficient $\mathrm{k}_{\mathrm{s}}$ such that $\mathrm{v}_{\mathrm{C}}=\mathrm{k}_{\mathrm{s}} \mathrm{m}_{\mathrm{C}}$ would be the strength of the localised skew quadrupole component. Once $k_{\mathrm{s}}$ is solved for, in a least square sense, its inverse can be incorporated into the back-propagation of the covariance matrix. If this corresponds to the dominant component of the beam coupling, then a drastic reduction in the off- 
diagonal elements should be seen upstream of this point. From this analysis no compelling evidence was found for a point source of coupling in the region from the beginning of TT2 to the beginning of TT10.

\subsection{Momentum dependence}

Measurements were also performed with the same optics but at $20 \mathrm{GeV} / c$. The results are shown in Table 4 . The 15-parameter fit resulted in non-physical momentum spread and high transverse-longitudinal correlation, probably reflecting marginal data redundancy. The uncoupled elements are quite reproducible while some fluctuation is observable in the coupled elements, probably as a consequence of the higher uncertainty on the dispersion at this momentum (it has been assumed that this is the same as measured at $26 \mathrm{GeV} / c$ ). A smaller beam coupling is nevertheless observed confirming the hypothesis of a coupling source in the PS ring or at extraction.

Table 4: Normalised Covariance Matrix at the beginning of TT10 for various fitting modes for a $20 \mathrm{GeV} / c$ transfer

\begin{tabular}{|c|c|c|c|c|c|}
\hline $\mathrm{X}$ & $X^{\prime}$ & Y & $Y^{\prime}$ & $\Delta \mathrm{P}$ & \\
\hline 0.002 & -0.877 & 0.043 & 0.072 & 0.140 & $\mathrm{X}$ \\
\hline & 0.0001 & -0.015 & -0.122 & -0.303 & $\mathrm{X}^{\prime}$ \\
\hline & & 0.0010 & 0.624 & -0.091 & $\mathrm{Y}$ \\
\hline & & & 0.00007 & 0.164 & $\mathrm{Y}^{\prime}$ \\
\hline \multicolumn{4}{|c|}{ 14-parameter fit $\left(\sigma_{p}=0.00025\right)$} & & $\Delta \mathrm{P}$ \\
\hline
\end{tabular}

\begin{tabular}{|c|c|c|c|c|c|}
\hline $\mathrm{X}$ & $\mathrm{X}^{\prime}$ & $\mathrm{Y}$ & $\mathrm{Y}^{\prime}$ & $\Delta \mathrm{P}$ & \\
\hline 0.002 & -0.866 & -0.108 & -0.028 & & $\mathrm{X}$ \\
\hline \multirow{7}{*}{} & 0.0001 & 0.125 & 0.030 & & $\mathrm{X}^{\prime}$ \\
\cline { 2 - 6 } & 0.0008 & 0.543 & & $\mathrm{Y}$ \\
\cline { 2 - 6 } & & 0.00008 & & $\mathrm{Y}^{\prime}$ \\
\cline { 2 - 6 } & & 0.00033 & $\Delta \mathrm{P}$ \\
\cline { 3 - 5 }
\end{tabular}

\begin{tabular}{|c|c|c|c|c|c|}
\hline $\mathrm{X}$ & $\mathrm{X}^{\prime}$ & $\mathrm{Y}$ & $\mathrm{Y}^{\prime}$ & $\Delta \mathrm{P}$ & \\
\hline 0.002 & -0.874 & -0.0089 & -0.0001 & & $\mathrm{X}$ \\
\cline { 2 - 6 } & 0.0001 & 0.018 & -0.0008 & & $\mathrm{X}^{\prime}$ \\
\cline { 2 - 5 } & 0.0009 & 0.526 & & $\mathrm{Y}$ \\
\cline { 2 - 5 } & 0.00008 & & $\mathrm{Y}^{\prime}$ \\
\cline { 2 - 5 } & &
\end{tabular}

\subsection{Improvements of the algorithm}

Two tasks are being carried out to improve the current technique and better understand its limitations:

- An algorithm has been implemented to perform noise subtraction: cut-off elliptical boundaries conforming to beam distribution are derived from pixel population without resorting to Gaussian fits (see contour lines in Fig. 1). Both the $\langle\mathbf{Y Y}\rangle$ and the normalised $\langle\mathbf{X Y}>$ correlations would be affected by $14 \%$ from rectangular cut-off for the case shown. The difference should be even more pronounced for a more tilted beam.

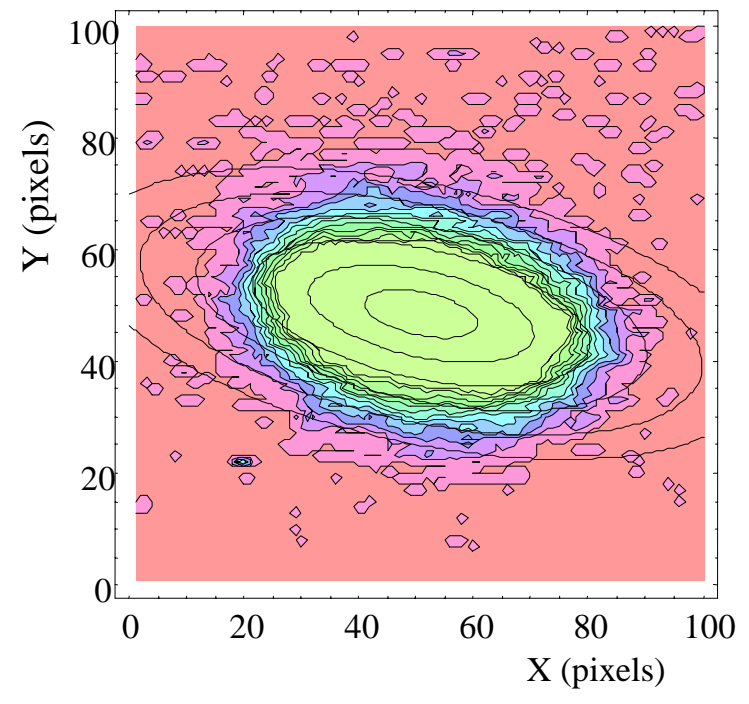

Figure 1: Measured beam distribution in $(\mathrm{X}, \mathrm{Y})$ space with noise cut-off based on elliptical boundaries (1 pixel corresponds to $170 \mu \mathrm{m}$ ).

- Information on errors on fitted quantities, as well as their covariance is needed. Fit weighting based on a conjectured formula for relative measurement errors yielded more physically consistent fitted beam covariance matrices and a momentum spread closer to the value measured in the PS. Rigorous physical justification for this conjecture is being studied.

- Condition analysis of the fitting system is needed. Questions such as relative sensitivity of fitted quantities to input and near singular combinations of parameters will be studied.

\section{CONCLUSIONS}

The measurement of the $5 \times 5$ beam covariance matrix from 2D OTR beam profile data was successfully applied to the PS/SPS transfer line, showing a significant betatron coupling for the LHC transfer at $26 \mathrm{GeV} / c$. Preliminary measurements performed at $20 \mathrm{GeV} / c$ show a less pronounced effect. This observation, together with the absence of compelling evidence for point sources of coupling in the transfer line as a result of independent analyses, seems to point to sources located upstream of the injection line, in the PS machine or at extraction.

\section{ACKNOWLEDGEMENTS}

We would like to thank the PS and SPS operation crews for assistance in the measurements and G. Ferioli, J. J. Gras, A. Guerrero and R. Jung for their help with OTR hardware and software.

\section{REFERENCES}

[1] G. Arduini et al, "Measurements and Optimisation of the PS-SPS Transfer Line Optics", PAC'99, New York, June 1999.

[2] Y. Chao, "New Results on TT2 TT10 matching", CERN-SL-2001-003. 